\title{
Evidence for variability timescale dependent UV/X-ray delay in Seyfert 1 AGN NGC 7469
}

\author{
Mayukh Pahari ${ }^{1}$, I. M. M ${ }^{\mathrm{c}}$ Hardy $^{1}$, Federico Vincentelli ${ }^{1}$, Edward Cackett $^{2}$, \\ Bradley M Peterson ${ }^{3,4}$, Mike Goad ${ }^{5}$, Kayhan Gültekin ${ }^{6}$, Keith Horne ${ }^{7}$ \\ 1 School of Physics 83 Astronomy, University of Southampton, Highfield campus, Southampton SO17 1BJ, UK \\ 2 Department of Physics and Astronomy, Wayne State University, 666 W. Hancock Street, Detroit, MI 48201, USA \\ 3 Department of Astronomy, The Ohio State University, $140 \mathrm{~W} 18^{\text {th }}$ Avenue, Columbus, OH 43210, USA \\ 4 Space Telescope Science Institute, 3700 San Martin Drive, Baltimore, MD 21218, USA \\ 5 University of Leicester, Department of Physics and Astronomy, Leicester, LE1 7RH, UK \\ 6 Department of Astronomy, University of Michigan, 500 Church Street, Ann Arbor, MI 48109, USA \\ 7 School of Physics and Astronomy, University of St. Andrews, Fife KY16 9SS, Scotland, UK
}

20 April 2020

\begin{abstract}
Using a month-long X-ray lightcurve from $R X T E / P C A$ and 1.5 month long UV continuum lightcurves from IUE spectra in 1220-1970 $\AA$, we performed a detailed time-lag study of the Seyfert 1 galaxy NGC 7469. Our cross-correlation analysis confirms previous results showing that the X-rays are delayed relative to the UV continuum at $1315 \AA$ by $3.49 \pm 0.22$ days which is possibly caused by either propagating fluctuation or variable comptonisation. However, if variations slower than 5 days are removed from the X-ray lightcurve, the UV variations then lag behind the X-rays variations by $0.37 \pm 0.14$ days, consistent with reprocessing of the X-rays by a surrounding accretion disc. A very similar reverberation delay is observed between Swift/XRT X-ray and Swift/UVOT UVW2, U lightcurves. Continuum lightcurves extracted from the Swift/GRISM spectra show delays with respect to X-rays consistent with reverberation. Separating the UV continuum variations faster and slower than 5 days, the slow variations at $1825 \AA$ lag those at $1315 \AA$ by $0.29 \pm 0.06$ days, while the fast variations are coincident $(0.04 \pm 0.12$ day). The UV/optical continuum reverberation lag from IUE, Swift and other optical telescopes at different wavelengths are consistent with the relationship: $\tau \propto \lambda^{4 / 3}$, predicted for the standard accretion disc theory while the best-fit X-ray delay from $R X T E$ and $S w i f t / X R T$ shows a negative X-ray offset of $\sim 0.38$ days from the standard disc delay prediction.
\end{abstract}

Key words: accretion, accretion disc — galaxies: Seyfert — black hole physics — X-rays: galaxies — galaxies: individual: NGC 7469

\section{INTRODUCTION}

Emission-line reverberation

mapping (Blandford and McKee 1982; Peterson 2014), based on measured lags between continuum and emission-line bands and the width of the emission line, is a very successful technique for determining AGN broad line region (BLR) size and black hole virial mass. Over 60 masses have currently been measured (Peterson et al. 2004; Bentz et al. 2009; Bentz and Katz 2015). Over the last 2 decades, considerable observational effort has also been put into continuum reverberation mapping, measuring the lags between a shortwavelength band, often the X-rays, and longer-wavelength UV and optical bands. The initial aim was to map the temperature structure of the accretion disc and hence find a standard candle by which distances could be estimated and the Hubble constant derived Cackett et al. (2007). Most such studies assumed a disc with the temperature structure as derived by Shakura \& Sunyaev (1973). Incident high energy emission will enhance the existing thermal emission leading to a wavelength $(\lambda)$ dependent lag, $\tau$, between the incident high energy, and re-radiated UV/optical emission, of $\tau \propto\left(M^{2} \dot{m}_{E}\right)^{1 / 3} \lambda^{\beta}$ where $\beta=4 / 3, M$ is the black hole mass and $\dot{m}_{E}$ is the accretion rate in Eddington units. Initial studies (Collier et al. 1999; Cackett et al. 2007) were consistent with $\beta=4 / 3$, but included only optical bands and did not extend to the X-ray bands.

Coordinated observations, usually with Rossi X-ray Timing Explorer (RXTE) (Arev́alo et al. 2009; Breedt et al. 
2009; Arev́alo et al. 2008; Marshall et al. 2008; Uttley et al. 2003; McHardy et al. 2003; Shemmer et al. 2003), and ground-based optical telescopes, mostly revealed a good correlation, with the optical lagging behind the X-rays, consistent with the expectations of reprocessing. However the lag measurements $\sim 1 \pm 0.5$ days were rarely statistically significant and could not rule out that the X-rays might lag behind the optical. These long $R X T E$-based programmes, which in some cases covered up to 10 years (Breedt et al. 2010), also showed that although there was a good correlation between the X-rays and the optical bands on short timescales (weeksmonths), on longer timescales (months-years) there were often trends in the optical lightcurves with no counterparts in the X-ray lightcurves.

Lags in the opposite sense, where the hard band lags the soft, are also seen on longer timescales. These hard lags have been seen in both X-ray binaries (from milliseconds to seconds) and AGNs (from days to months) (Papadakis et al. 2001; McHardy et al. 2004; Arev́alo and Uttley 2006), and are thought to arise due to the inward propagation on viscous timescales of mass accretion fluctuations in the disc that are then transmitted to the corona (Kotov et al. 2001; Arev́alo and Uttley 2006; Uttley et al. 2011).

More intense, multi-band, observations with Swift (Shappee et al. 2014; McHardy et al. 2014; Edelson et al. 2015; Fausnaugh et al. 2016; Troyer et al. 2016; Edelson et al. 2017; McHardy et al. 2018; Pal and Naik 2018; Edelson et al. 2019) confirmed the general picture of wavelength dependent UV/optical lags, consistent with disc reprocessing, although the measured lags were $\sim 2-3$ times longer than expected theoretically (McHardy et al. 2014). This discrepancy may indicate an inhomogeneous disc (Dexter \& Agol 2011). The Swift observations also provide evidence of reprocessing of high energy emission from a larger reprocessor than just the accretion disc, probably the broad line region (BLR) clouds (Korista and Goad 2019; Chelouche et al. 2019; Lawther et al. 2018; McHardy et al. 2018; Cackett et al. 2018; Sun et al. 2018; Pal and Naik 2018). This evidence is in the form of an excess lag in the U-band (Edelson et al. 2017; Fausnaugh et al. 2016; Edelson et al. 2015), which contains the Balmer continuum, (Kotov et al. 2001) and an excess lag at $3634 \AA$ (known as the Balmer jump), and also in the fact that the reprocessing function required to explain the optical emission as reprocessing of X-ray emission, has a tail to long delays (a few days) as well as a sharp peak at short timescales ( $\sim$ hours) from the disc. The Swift observations also show that although wavelength-dependent lags following roughly $\tau \propto \lambda^{4 / 3}$ apply in most AGN between the UV and optical bands, the lag between the X-ray and UV band is usually much larger than expected purely from extrapolation of the UV-optical lag spectrum down to X-ray wavelengths (Dai et al. 2010; Morgan et al. 2010; Mosquera et al. 2013; Edelson et al. 2015; Fausnaugh et al. 2016; McHardy et al. 2018). Moreover, the X-ray/UV correlation is weaker than the UV/optical one (Edelson et al. 2019).

There are a number of possible explanations for this increased lag, including that the lag corresponds to the thermal timescale due to the thermal reverberation from a significantly hot disc with low accretion rate (Kammoun et al. 2019; Sun et al. 2018), hot accretion flow with a disc truncation (Noda et al. 2016), very large area of the reprocessing site (Pal et al. 2017; Pal and Naik 2018), non-blackbody nature of the emerging disc spectra due to the low atmospheric density (Hall et al. 2018). Other potential explanations include that the X-rays do not directly illuminate the outer disc but are first reprocessed by, and scattered through, the scattering atmosphere (Narayan 1996), the inflated inner edge of the accretion disc, which introduces an additional lag (Gardener and Done 2017).

The increase in X-ray/UV-optical correlation strength on short timescales, originally noted by Breedt et al. (2009), has also been noted in Swift observations. For example in NGC 5548 when variations on timescales longer than 20 days are removed from UV lightcurves, the correlation improves and, moreover, the X-ray to UV lag now falls on an extrapolation of the UV-optical lag spectrum. A similar behaviour is seen in NGC 4593 (McHardy et al. 2018) where the Xray/UV lag decreases when long timescale variations $(>10$ days), presumably from the BLR, are removed from the UV lightcurves. The resultant X-ray/UV lag is then again in agreement with an extrapolation of the UV-optical lag spectrum to X-ray wavelengths.

The one notable exception to the general scenario that the UV/optical variations on short timescales are mainly driven by reprocessing of high energy (i.e. X-ray) variations is NGC 7469, a bright, infrared luminous, Sb-type spiral Seyfert 1 galaxy at a red-shift of 0.016268 . The mass of the central supermassive black hole is $9.04_{-0.97}^{+1.06} \times 10^{6} \mathrm{M}_{\odot}{ }^{1}$ (Peterson et al. 2014; Zu et al. 2011).

In June-July 1996 NGC 7469 was observed almost continuously, Earth occultations excepted, for a period of $\sim 46$ days by $I U E$, providing UV spectra from which lightcurves can be produced in a variety of bands (Wanders et al. 1997). For 30 of these days there was almost continuous $R X T E / \mathrm{PCA}$ monitoring. Both the IUE and $R X T E$ lightcurves are dominated by a small number of large amplitude, quasi-sinusoidal, variations with peak to peak timescales of around 15-20 days (see Figure 2 of Nandra et al. 1998). Nandra et al. (1998) show that the peaks in the UV lightcurve led the peak in the X-rays by $\sim 4$ days. Although there are only 2 cycles of variability in this analysis, these observations nonetheless led to much speculation regarding physical mechanisms which might explain the 4d X-ray lag. Nandra et al. (1998) suggested that the $\mathrm{X}$-rays might be produced by up-scattering of UV photons by a variable coronal structure. Using the same UV/X-ray observations, Petrucci et al. (2004) performed UV and hard $\mathrm{X}$-ray joint spectral fitting and found an anti-correlation between the UV flux and the X-ray coronal temperature. The explanation of such an anti-correlation requires strong variability in coronal structure over days rather than a simple disc-corona structure (Petrucci et al. 2004).

However, just within the IUE band, Wanders et al. (1997) estimated the delay of Ly $\alpha$, C IV, N v, Si IV and He II emission lines with respect to the UV continuum as 2.3-3.1 days, $\sim 2.7$ days, 1.9-2.4 days, 1.7-1.8 days and 0.7-1 day respectively which is broadly consistent with observations

1 This neglects a further $\sim 0.4$ dex uncertainty due to using the population mean value of the dimensionless factor $f$, which depends on the uncertain geometry and orientation of the BLR in each object. 
IUE spectra of NGC 7469

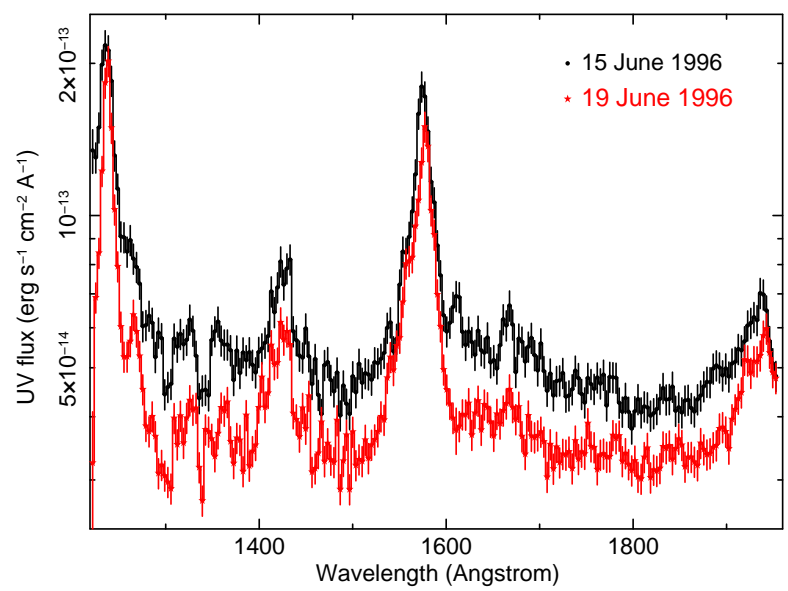

IUE UV spectrum fitted with continuum and line models

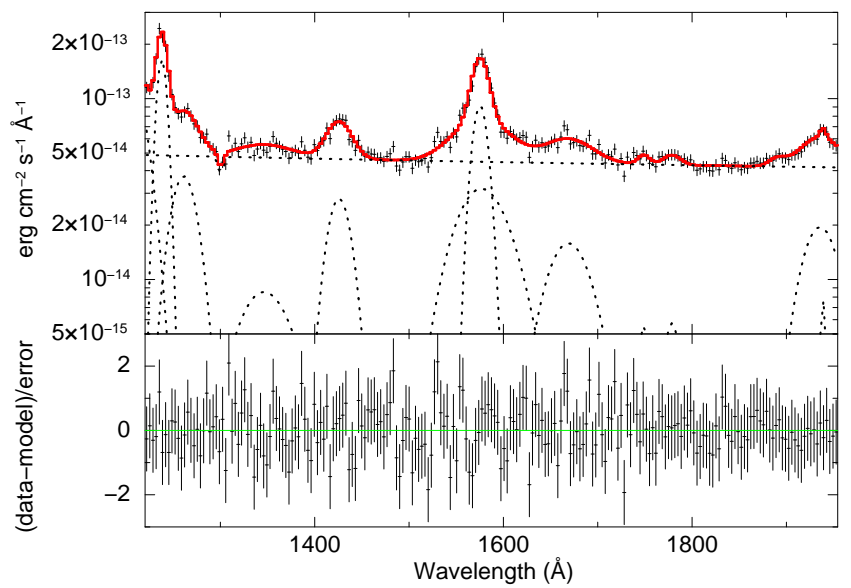

Figure 1. UV variability of NGC 7469 and spectral modelling: Left: IUE spectra of the NGC 7469 nucleus in the wavelength range of 1220-1970 $\AA$ as observed on 15 June 1996 (circles) and 19 June 1996 (stars) respectively. A substantial decrease is observed in the UV continuum flux level within 4 days. Right: the best-fit time-averaged UV spectrum (1220-1970 A) from IUE fitted with a model (red) consisting of a continuum, broad and narrow emission features and narrow absorption (dotted lines; top) and the residual of the fitting (bottom). Each of 218 spectra is fitted separately with the best-fit model to obtain the UV continuum and line fluxes from each pointing.

of other Seyfert galaxies. Using concurrent ground-based spectro-photometric monitoring of NGC 7469, Collier et al. (1998) found that continuum variations at $4865 \AA$ and 6962 $\AA$ lag those at $1315 \AA$ by $1.0 \pm 0.3$ days and $1.5 \pm 0.7$ days respectively. They also noted that the continuum variations at $1485-1825 \AA$ lag those at $1315 \AA$ by $0.21-0.35$ days, which is consistent with the expectations from disc reprocessing. Later, using a more sophisticated spectral modelling approach, using the HST/FOS spectrum as a template, Kriss et al. (2003) found that the continuum variations at $1485 \AA, 1740 \AA$ and $1825 \AA$ is delayed relative to the shorter UV continuum at $1315 \AA$ by $0.09,0.29,0.36$ days respectively, again in good agreement with disc reprocessing.

The main remaining unexplained problem, therefore, is the relationship between the X-ray and UV variations. In this paper we re-examine the relationship between the X-ray and UV variations as observed by $R X T E$ and IUE. In addition to examining the correlation in the raw lightcurves, we also search for a correlation in lightcurves from which the long timescale, large amplitude, variations have been removed (Section 3). Here we find lags that are more consistent with the reprocessing scenario (Section 4.1). We also examine archival Swift data which, although with considerably greater uncertainties than in the $I U E$ data, allow us to extend our lag measurements into the optical bands (Section 5). These data also allow us to determine whether the apparent lag of the UV by the X-rays seen with IUE and $R X T E$, when considering long timescale variations, is a common phenomenon. This does not appear to be the case. We compare the observed wavelength-dependent lag with that predicted We conclude the paper (Section 7) with a brief summary of the observational results and with some general overall interpretations.

\section{OBSERVATION}

RXTE performed 311 observations of NGC 7469 between
10 June 1996 00:44:16 and 11 July 1996 23:59:19. For each observation, we extract the $R X T E / \mathrm{PCA}$ lightcurve in the 2$15 \mathrm{keV}$ energy range, combining observations from PCU0, PCU1 and PCU2 which were operational during the entire period of the observations. IUE continuously monitored NGC 7469 between 1996 June 10 and July 29 producing in total of 218 low-dispersion UV spectra in the wavelength range of 1150-1975 A. The details of the analysis procedure are provided in Wanders et al. (1997). Raw images were processed using the TOMSIPS (Ayres 1993) and NEWSIPS (Nichols et al. 1993) data reduction packages. In this work, we consider NEWSIPS pipeline reduced spectra as mentioned by Wanders et al. (1997), a nonlinear wavelength calibration error exists in TOMSIPS reduction since long-term drifts in the wavelength scale were not taken into account. On the other hand, NEWSIPS reduced spectra matches well with the HST spectra without any corrections applied. We also use data from Swift and the Wise Observatory optical telescope which are described later. Details of observations which are used to perform continuum reverberation mapping are provided in Table 1.

\section{DATA REDUCTION}

Lightcurve extractions are performed using Heasoft 6.25 packages applying standard filtering criteria. Further details on the observation and analysis procedures are provided by Nandra et al. (1998).

In case of NEWSIPS pipeline reduced IUE spectra, due to a small shift (1-2 $\AA$ ) in wavelength caused by the large aperture pointing errors (Wanders et al. 1997), an offset compensation is performed so that the sharp C IV line feature of all spectra falls at same average wavelength. Extinction corrections are not significant due to the very low interstellar reddening $\mathrm{E}(\mathrm{B}-\mathrm{V})=0.059$ and background corrections are applied. The red-shift observed from the C IV average peak is consistent with the spectroscopic red-shift of 
X-ray and UV continuum lightcurve of NGC 7469

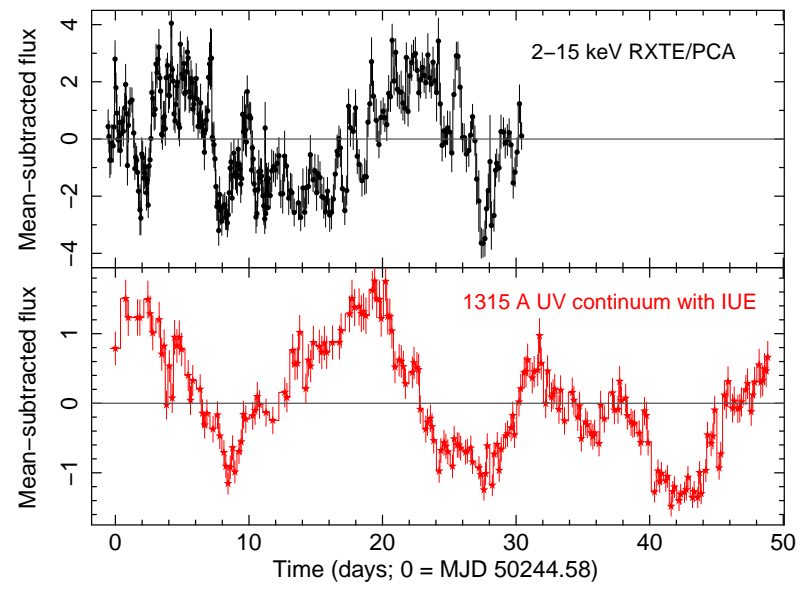

UV/X-ray cross-correlation

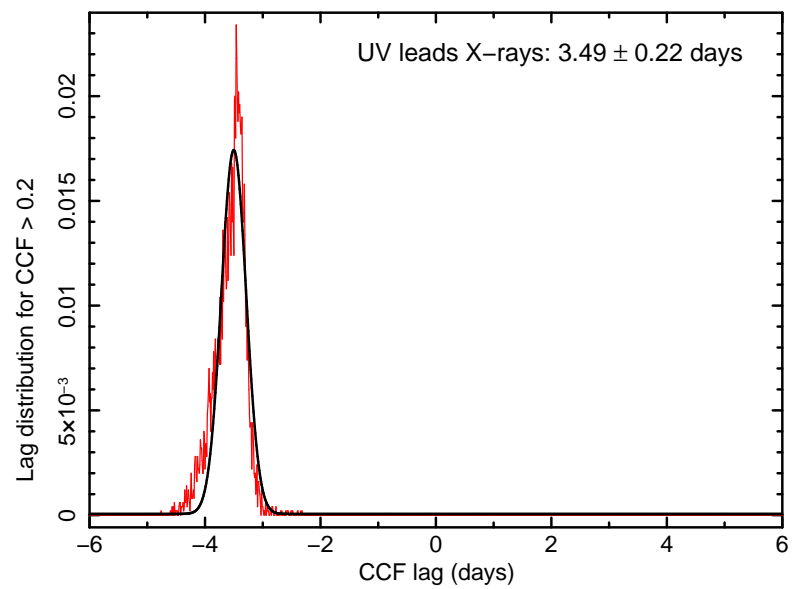

Figure 2. $X$-ray/UV cross-correlation: Left: $2-15 \mathrm{keV}$ background-subtracted $R X T E / \mathrm{PCA}$ lightcurve (top) and rest-frame $1315 \AA$ UV continuum lightcurve observed with $I U E$ (bottom). Both lightcurves are mean-subtracted for a better visibility of the relative flux variability. When both lightcurves are cross-correlated using Monte Carlo-based combined flux randomisation and random subset selection methods(FR/RSS), the relative frequency distribution of the cross-correlation function centroids (CCF; when cross correlation coefficient $>0.2)$ is shown in the right panel. The lag distribution is fitted with a Gaussian. The centroid and the FWHM/2 of the Gaussian with stronger peak is quoted as the time delay and its uncertainty, respectively.

X-ray and UV fast varibility in NGC 7469

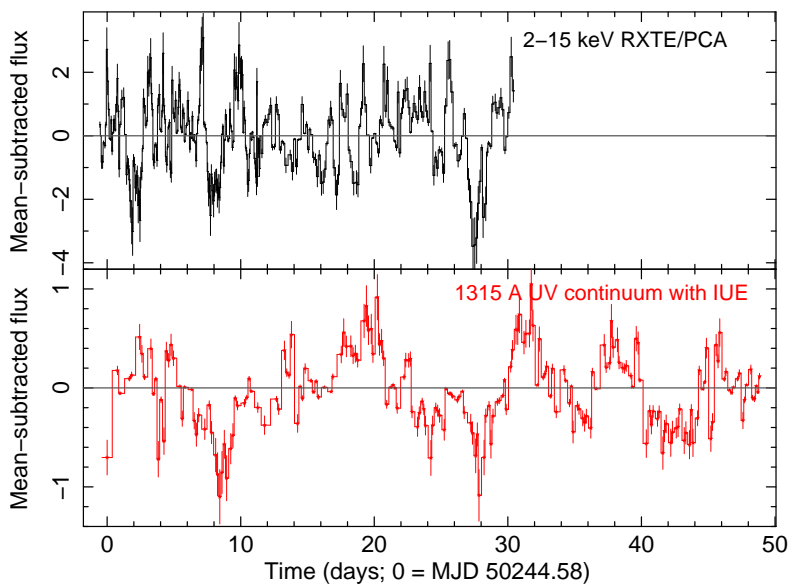

Filtered X-ray/UV cross-correlation

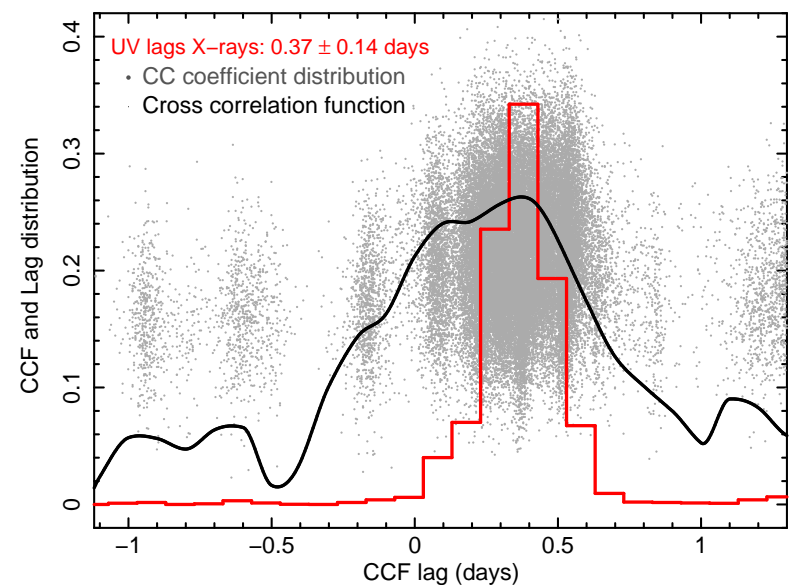

Figure 3. The effect of variability filtering: After filtering the variability longer than 5 days from X-ray and UV lightcurves shown in the left panel of Figure 2, the resulting lightcurves are shown in the left panel. When these filtered lightcurves are cross-correlated using the $\mathrm{MC}$ based flux randomisation and random subset selection methods combined (FR+RSS), the resulting frequency distribution is shown in the right panel where a switch in lag is observed at a timescale comparable to the accretion disc reverberation timescale. The solid line shows the interpolated cross correlation function (CCF). The grey dots are the centroid lags from all of the FR+RSS MC based centroid lag calculations and the histogram give the $\tau_{c e n t}$ distribution for dots above the adopted threshold, CCF $>0.2$.

$z=0.00163$ from the $[\mathrm{OIII}]$ line at $5007 \AA$ (Salamanca et al. 1995).

\subsection{UV spectral fitting}

IUE UV spectra show significant variability in the continuum flux level. An example of such variations is shown in the left panel of Figure 1. At all wavelengths between 1220-1970 A, the continuum UV flux as observed on 1996 June 15 is significantly higher than that on 1996 June 19. To study flux variability, we extract lightcurves at different wavelengths. To extract UV continuum and line lightcurves from UV spectra, we adopt a slightly different approach than
Wanders et al. (1997). Using the $\chi^{2}$ minimisation technique in XSpec, we fit the average spectrum with suitable combinations of a powerlaw function that represents the underlying continuum and multiple narrow and broad Gaussian components that describe emission lines. The best-fit model yields residuals with $\chi^{2}$ per degrees of freedom $=179 / 175$. The right panel of Figure 1 shows the time-averaged UV spectrum fitted with different model components and the residual of the fitting. The powerlaw energy spectral index from the best-fit average UV spectrum is observed to be $1.67 \pm 0.11$ which is consistent with that typically observed from AGN (Shull et al. 2012) and also consistent with the radio-quiet nature of NGC 7469 (Baldi et al. 2015). Using 
X-ray (filtered) and UV continuum cross-correlation

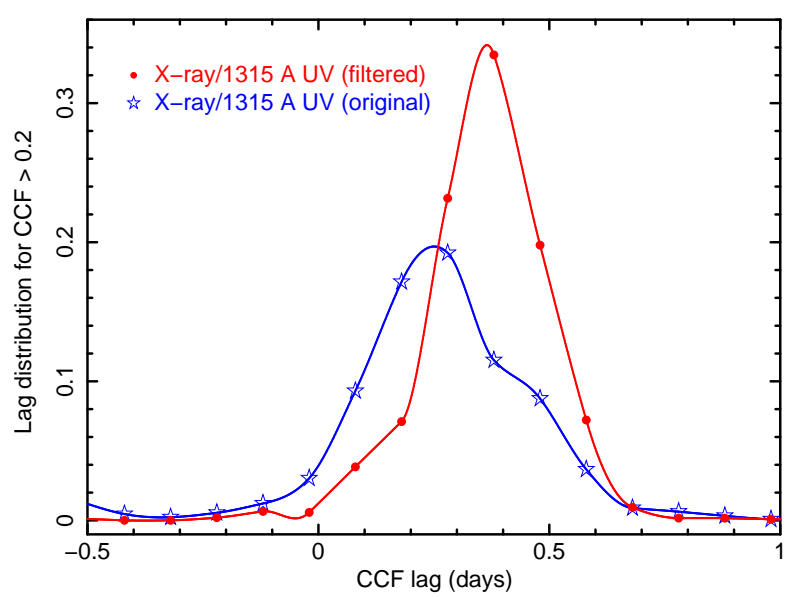

X-ray and UV lag centroid distribution from auto-correlation

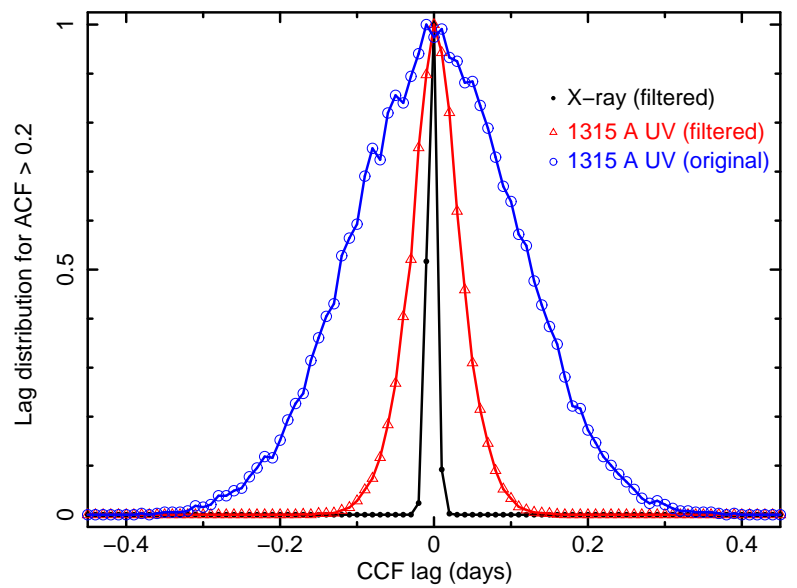

Figure 4. Effect of filtering on $X$-ray/UV cross correlation: Left panel shows the lag distribution for FR/RSS samples with centroid $\mathrm{CCF}>0.2$ when the hi-pass filtered X-ray lightcurve is cross-correlated with the filtered UV continuum at $1315 \AA$ (circles) and with the unfiltered UV continuum (stars) using the flux randomisation (FR) and random subset selection (RSS) methods combined. A significant overlap between the two distributions can be observed. Right panel shows auto-correlation distribution function of the filtered X-ray (filled circles) and both filtered (triangles) and unfiltered (empty circles) UV continuum at $1315 \AA$. Both filtered and unfiltered UV auto-correlation functions are wider than filtered X-ray auto-correlation function.

the best-fit model, we fit individual $I U E$ spectra by fixing the continuum powerlaw index and letting all other parameters vary. From individual spectrum fitting, we derive spectral line and continuum parameters such as line width and flux. One advantage of using spectral modelling over numerical integration is that line and continuum fluxes can be measured more accurately, particularly when narrow/broad lines are close to each other and there exists an underlying broad continuum. For example, Vestergaard \& Wilkes (2001) showed that the asymmetry in the blue wing of the C IV line is caused by the presence of high-ionization Si II lines at $\sim 1540 \AA$. Therefore, the use of two Gaussian components at both line locations provide more accurate modelling and hence flux measurements of the underlying continuum. From the best-fit model, continuum UV flux is computed in the rest-frame wavelength range of 1306-1327 $\AA$, 1473-1495 $\AA, 1730-1750 \AA$ and 1805-1835 $\AA$ using a convolution model (cflux in XSpec) that provides integrated, continuum-subtracted flux over a given wavelength range and its $1 \sigma$ error. Wavelength ranges for continuum flux measurement are kept consistent with those from Wanders et al. (1997).

\section{$3.2 \quad$ Filtering}

In this paper we are searching for signs of reprocessing in the original RXTE and IUE observations, which are dominated by a very small number ( 2 in the X-ray observations, 3 in the IUE campaign) of large amplitude variations with peak to trough timescales of $\sim 10$ days. If NGC 7469 behaves like other AGN of similar mass and accretion rate, the reprocessing signature should be manifest by the UV lagging the Xray lightcurves by less than a day. It is well established that long-term variations can distort the measurement of shortterm lags in CCFs (Welsh 1999) and so we filter out the long timescale, large amplitude, variations. We choose a 5-day filtering timescale which will eliminate variations on longer timescales but will allow lags on timescales shorter than 5 days, of both positive (i.e., reprocessing) and negative (seed photon variation) sign, to be detected. Similar filtering techniques have been used successfully to reveal short timescale correlations in other AGN, eg NGC 5548 (McHardy et al. 2014) and NGC 4593 (McHardy et al. 2018).

To filter the lightcurves, we use a locally-weighted scatter plot smoothing (LOWESS) function which is based upon a non-parametric, non-linear least square regression method (Cleveland and Devlin 1988). The weight function used for LOWESS is the tricube kernel function: $k(d)=\left(1-|d|^{3}\right)^{3}$ where $d$ is the distance of a given data point from the point on the curve being fitted, scaled to lie in the range from 0 to 1 . For the filtering purpose, $d(t) \equiv\left(t-t_{i}\right) / \Delta t$ is the time difference between time $t$ and the data point $i$ at time $t_{i}$ in units of $\Delta t=5 \mathrm{~d}$. Such a function has higher efficiency than traditional kernel functions like boxcar or triangular and does not require specification of the model function to fit the data, therefore, making it ideal to fit complex processes where no theoretical model exists. When compared to the efficiency of the Epanechnikov kernel function, the relative efficiency of the LOWESS kernel is $99.8 \%$ while the same for the boxcar and triangular kernels are $92.9 \%$ and 98.6\% respectively (Epanechnikov 1969). The efficiency of a function $\mathrm{f}(\mathrm{x})$ is defined as $\sqrt{\int x^{2} f(x) d x} \int f(x)^{2} \mathrm{~d} x$.

In this work, the LOWESS filter is used (residual from the LOWESS function fitting) to eliminate variability slower than 5 days in the X-ray and UV lightcurves. In the rest of the paper and in all Figures, the word 'filtered' implies a 5 -day filtering unless otherwise specified.

\subsection{Correlation and delay measurements}

Since X-ray and UV observations have dissimilar temporal coverage, we use the interpolated cross-correlation function (CCF) to compute the delay among X-ray and various UV 


\begin{tabular}{|c|c|c|c|}
\hline $\begin{array}{l}\text { Telescope/ } \\
\text { Instrument }\end{array}$ & $\begin{array}{l}\text { Observation } \\
\text { time (MJD) }\end{array}$ & $\begin{array}{l}\text { no. of exposures } \\
\text { /data points }\end{array}$ & $\begin{array}{c}\text { Wavelength } \\
\text { coverage }(\AA)\end{array}$ \\
\hline RXTE/PCA & $50244-50275$ & 311 & $0.83-6.22$ \\
\hline IUE & 50244-50294 & 218 & $1150-1975$ \\
\hline _Swift/XRT & 54630-58230 & 176 & $1.24-24.8$ \\
\hline$\overline{\mathrm{S}} \overline{\mathrm{sift}} / \overline{\mathrm{U}} \overline{\mathrm{V}} \overline{\mathrm{OT}} \overline{\mathrm{T}}$ & & & \\
\hline$U V W 2$ & 54644-58229 & 98 & $1928 \pm 657$ \\
\hline$U$ & $54635-57423$ & 61 & $3465 \pm 785$ \\
\hline$U V-G R I S M$ & $56410-56524$ & 37 & $1700-2900$ \\
\hline${ }^{\bar{c}} \overline{\mathrm{W}}$ ise $\overline{\mathrm{F}} \overline{\mathrm{O}} \overline{\mathrm{SC}} \overline{\mathrm{C}}^{-}$ & $5 \overline{0} \overline{3} \overline{7}-5 \overline{0} 2 \overline{9} 5$ & $\overline{4}$ & $\overline{4} \overline{0} \overline{1} \overline{6}-\overline{7} \overline{4} \overline{1}$ \\
\hline
\end{tabular}

Table 1. Details of observations from different telescopes and satellites used in the present work. Central wavelengths and FWHM (Poole et al. 2008) are quoted in case of UVW2 and U filters.

continuum and line lightcurves. Uncertainties on lag measurements are computed using a Monte Carlo simulation to assess the flux uncertainties associated with each measurement and the sampling uncertainties of the observed time series, similar to bootstrapping. The details of the implementation of both methods are provided in Peterson et al. (1998) and denoted as the flux randomisation (FR) and random subset selection (RSS) methods respectively. For each Monte Carlo realisation of a light curve with $\mathrm{N}$ data points, $\mathrm{N}$ selections are drawn at random (i.e., random subset selection or RSS); for data points that are randomly selected multiple times $M$, the associated uncertainty is decreased by $M^{1 / 2}$. The data points are then altered by adding random Gaussian deviates with a dispersion equal to the assigned flux uncertainty (i.e., FR). Multiple realisations result in a distribution of cross-correlation functions, and the corresponding joint distribution of CCF peak correlation coefficients and centroid lags. Centroid lag $\left(\tau_{\text {cent }}\right)$ is computed by averaging lag over regions where CCF is above $80 \%$ of the peak.

We perform 100,000 FR+RSS simulations for each pair of X-ray/UV lightcurves in this work and consider only measurements for which the cross-correlation coefficient is higher than 0.2. Although the resultant lag distribution may deviate from the normal distribution, we fit the resulting CCF centroid distribution using a Gaussian function and interpret the Gaussian centre as the measured time delay and its half width at half maxima $(\mathrm{FWHM} / 2)$ as the uncertainty on the delay measurements.

\section{ANALYSIS AND RESULTS}

The left panel of Figure 2 shows the 2-15 keV X-ray lightcurve (top) and the 1306-1327 $\AA$ UV continuum lightcurve (bottom; referred here $1315 \AA$ band) observed with $R X T E$ and IUE respectively. Both lightcurves are shown as residuals after subtracting the mean flux for comparison of lightcurve variability relative to their mean value. The right panel shows the centroid lag distribution of the FR+RSS cross-correlation between the X-ray and UV continuum lightcurves. The cross-correlation distribution peak indicates that $\mathrm{X}$-rays are delayed relative to the UV $1315 \AA$ band continuum by $3.49 \pm 0.22$ days. This is con- sistent with previous measurements by Nandra et al. (1998) but inconsistent with reprocessing models in which the UV variations should lag the $\mathrm{X}$-rays.

\subsection{Effect of lightcurve filtering}

The filtered X-ray and UV lightcurves are shown in the left panel of Figure 3. Visually the lightcurves are now quite similar. An FR+RSS cross-correlation between filtered lightcurves along with the cross correlation function, shown in the right panel of Figure 3, clearly demonstrates that on a timescale faster than 5 days, the UV continuum lags the $\mathrm{X}$-rays by $0.37 \pm 0.14$ days. The solid black line in the right hand panel of Figure 3 shows the interpolated cross correlation function (White \& Peterson 1994) for the lightcurves in the left hand panel of Figure 3. The grey dots are the centroid lags from all of the FR+RSS MC based centroid lag calculations based on these same lightcurves. The histogram is the distribution of $\mathrm{MC}$ centroid lags selecting only those where peak CCF value is $>0.2$. A similar lag is obtained when we truncate the UV lightcurve to match the duration of the X-ray lightcurve. Such a lag timescale is consistent with the accretion disc reverberation delay observed from other AGN (see sect. 5 and 6 for details). Such a switch in the sign of the lag, as well as the change in lag timescale, is remarkable.

To check the effect of UV filtering on the measured cross-correlation with X-rays, we perform the FR+RSS cross-correlation between the filtered X-ray and unfiltered UV lightcurve and plot the CCF lag distribution in the left panel of Figure 4 along with the X-ray/UV CCF centroid distribution with the filtered UV continuum. Clearly, X-ray and UV CCF centroid distributions using both filtered and unfiltered UV continuum significantly overlap with each other. Such an overlap implies that both fast and slow UV variability from hours to days timescale is mostly driven by fast X-ray variability.

To explore the connection between the driving and driven variability further, we perform auto-correlation analysis using filtered X-ray, filtered and unfiltered UV lightcurves. The FR+RSS method and the auto-correlation distribution is shown in the right panel of Figure 4. The lag centroid distribution of all three ACFs are well defined. However, the X-ray auto-correlation is significantly narrower than both filtered and unfiltered $1315 \AA$ UV autocorrelation. Such characteristics indicate that the UV variations are smoother than that of the X-ray variability, causing a wider ACF lag distribution for UV than for X-rays.

To test whether the delays among different UV bands depend on wavelength, we carry out a further check. We consider $1315 \AA$ and $1825 \AA$ (integrated flux in 1805-1835 $\AA$ ) band continuum lightcurves shown in the left panel of Figure 5. Both lightcurves have similar coverage, the same number of data points and the variability at different timescales is most likely driven by similar physical processes. Both lightcurves are mean-subtracted residuals and similar y-axis scales are used for an easy visual comparison of their variability. A cross-correlation between both continuum bands indicates that $1825 \AA$ continuum lags behind the $1315 \AA$ continuum by $0.34 \pm 0.17$ days, as shown in the right panel of Figure 5. We apply the filter to both lightcurves, decomposing them into slow and fast components separated 
UV continuum lightcurve with IUE

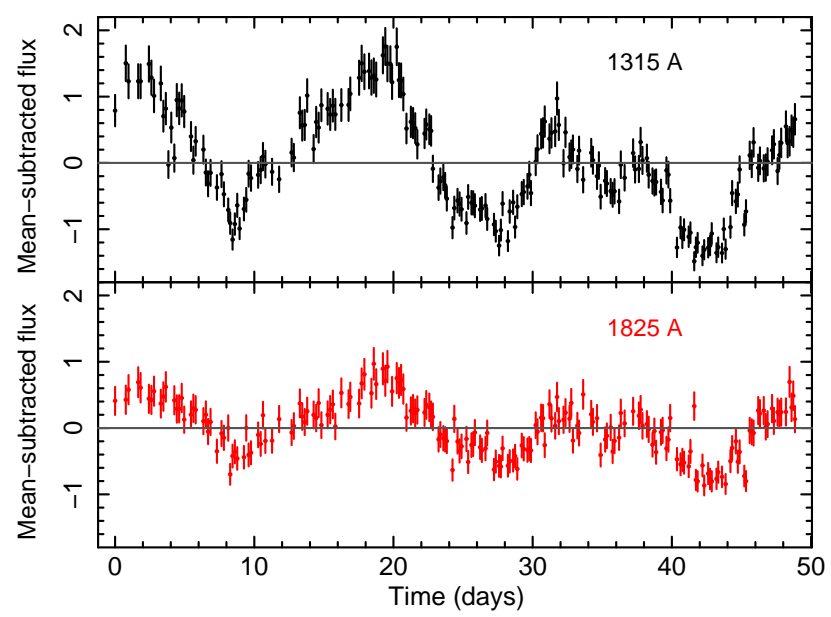

1315 A UV/1825 A UV cross-correlation

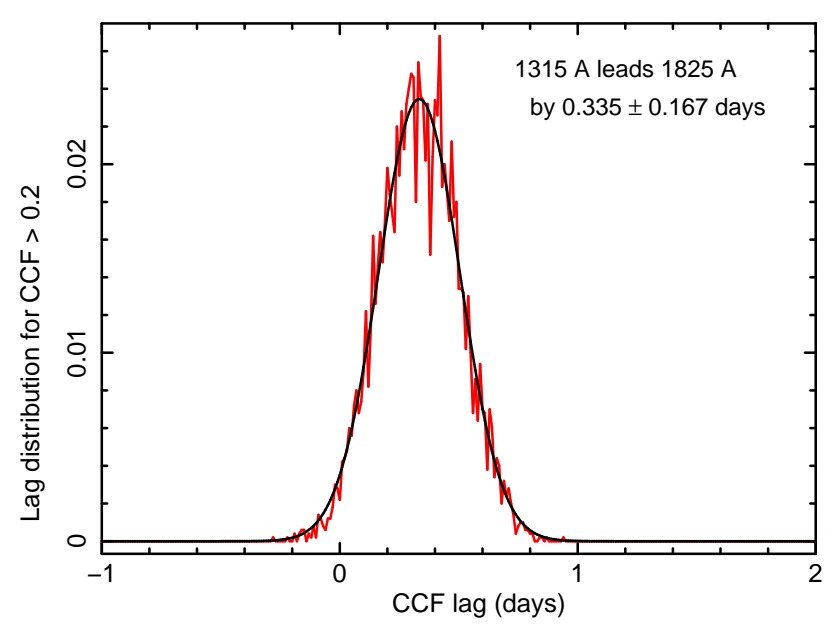

Figure 5. UV continuum lightcurve and CCF: Left panel shows $1315 \AA$ (top) and $1825 \AA$ (bottom) UV continuum lightcurves from NGC 7469. Both lightcurves are mean-subtracted residuals and similar y-axis scales are used for easy comparison of their flux variability. When they are cross-correlated using FR+RSS method, the distribution of centroid lags (for CCF > 0.2) is shown in the right panel. The frequency distribution is fitted with a Gaussian whose centroid and the FWHM/2 are quoted as the time delay and its uncertainty respectively.

$1315 \mathrm{~A}$ (fast) / $1825 \mathrm{~A}$ (fast) cross-correlation

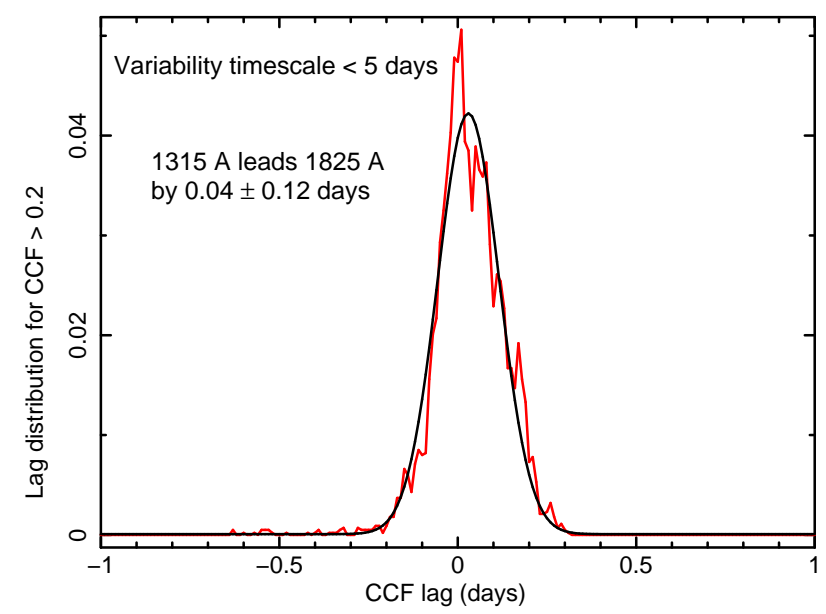

1315 A (slow) / 1825 A (slow) cross-correlation

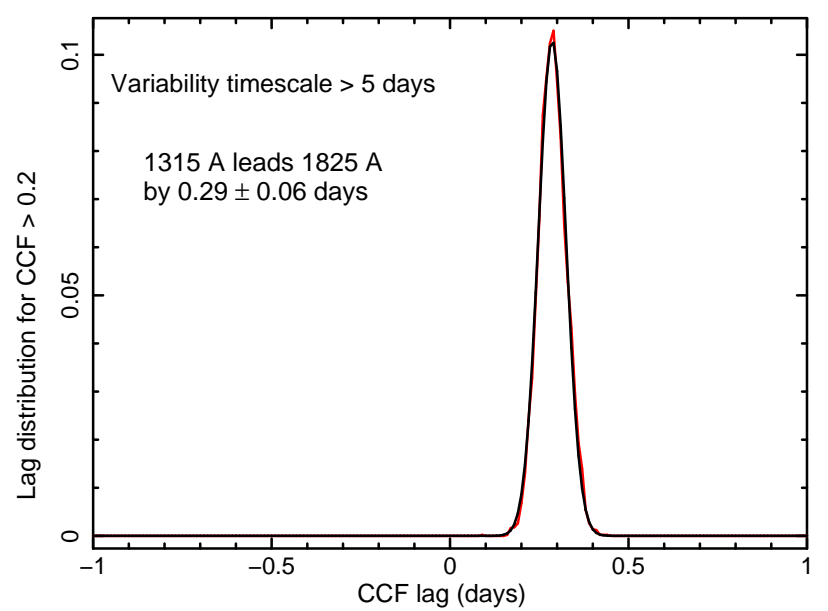

Figure 6. The effect of lightcurve filtering in UV: Left panel shows relative frequency distribution of CCFs computed with $1315 \AA$ and $1825 \AA$ UV continuum lightcurves (left panel of Figure 5) after applying a slow filter which only preserve fast (faster than 5 days) variability. On the other hand, when a fast filter is applied which only preserve slow (slower than 5 days) variability, the resulting CCF is shown in the right panel. Both distributions is fitted with a Gaussian whose centroid and FWHM/2 are quoted as the time delay and its uncertainty respectively. A significant difference between the fast and slow variability delay measurements can be noted.

at a 5-day timescale. The cross-correlation between the $1315 \AA$ and $1825 \AA$ fast lightcurves using FR+RSS technique yields that the $1825 \AA$ fast variability lags behind the $1315 \AA$ A fast variability by $0.04 \pm 0.12$ days, consistent with zero lag and shown in the left panel of Figure 6. However, when the $1315 \AA$ slow variability is cross-correlated with the $1825 \AA$ slow variability, the resulting distribution indicates that $1825 \AA$ band is delayed to the $1315 \AA$ band by $0.29 \pm$ 0.06 days (shown in the right panel of Figure 6). Therefore, the slower variability is significantly more delayed than the faster variability in the longer UV continuum.

\section{SWIFT MONITORING AND UV/OPTICAL LAG MEASUREMENTS}

The log of Swift observations is given in Table 1. There are 176 XRT photon counting X-ray $(0.5-10 \mathrm{keV})$ visits. Most of the X-ray observations were accompanied by UVOT imaging mode UVW2 (1928 $\AA$ ) observations with a lesser number including U (3465 $\AA$ ) observations. Some UVOT observations employed the GRISM. X-ray and UV flux measurements were performed using the Southampton pipeline ${ }^{2}$ (McHardy et al. 2018) based on standard Swift data analy-

2 https://swiftly.soton.ac.uk/ 
sis procedures (Cameron et al. 2012). The X-ray, UVW2, U filter lightcurves of NGC 7469 are shown in the top left panel of Figure 7. On two occasions, Swift observed NGC 7469 continuously over a period of nearly 4 months in X-ray, UVW2 and $\mathrm{U}$ filters. One such set of observations taken between MJD 56400 and MJD 56550 is shown in the top right panel of Figure 7. We have also extracted continuum lightcurves from 37 observations taken using Swift/GRISM between 28 April and 20 August 2013. Using GRISM data, continuum fluxes are measured at $2150 \AA, 3100 \AA$ and $4600 \AA$ respectively, and corresponding lightcurves are shown in the bottom left panel of Figure 7. For each UVOT filter and each continuum lightcurve from GRISM, we perform FR+RSS cross correlation with the X-ray lightcurve to measure the wavelength dependent lag. The bottom right panel of Figure 7 shows the $\mathrm{CCF}$ and corresponding lag distribution computed for X-ray and UVW2 filter (top) and for the X-ray and GRISM (bottom), respectively. We performed a wavelength-dependent cross correlation study between the X-ray and UVW2, U filter lightcurves using the FR/RSS technique. With respect to the X-rays, we found that the UVW2 and U filter lightcurves are delayed by $0.72 \pm 0.51$ days and $1.57 \pm 0.71$ days, respectively. These delays are shown by empty stars in the left panel of Figure 8 while the X-ray point is shown by the solid star. Along with the Swift measurements, for comparison, we include the continuum UV delay from IUE (shown by empty circles). The lag uncertainty merely reflect the number of data points and the measurement errors in the relevant lightcurves.

We performed the FR+RSS cross-correlation between $2150 \AA$ and $3100 \AA$ and between $2150 \AA$ and $4600 \AA$ lightcurves respectively and measured the delay. To verify and confirm our results, we repeat the similar exercise of the delay measurement by replacing the $2150 \AA$ lightcurve with the simultaneous Swift/UVW2 lightcurve. They are similar to within the measurement uncertainties. The Resulting delays with respect to X-rays are shown by the triangles in Figure 8. To compare fluxes during different campaigns, we compute and plot the average flux density at different wavelengths in the right panel of the Figure 8. Flux densities at similar wavelengths are consistent during different campaigns.

\subsection{Optical continuum from FOSC spectroscopic camera}

During the RXTE and IUE joint campaign in 1996, NGC 7469 was also monitored using the Faint Object Spectroscopic Camera (FOSC) mounted on the $1 \mathrm{~m}$ optical telescope at the Wise Observatory, Tel Aviv University (Kaspi et al. 1996; Collier et al. 1998). Between 02 June and 30 July 1996, 42 spectroscopic observations were taken with a spectral resolution of $\sim 6$ Ain the wavelength range 4016$7841 \AA$ (Collier et al. 1998). While the analysis details and lightcurves are provided by Collier et al. (1998), the optical continuum lightcurves at $4845 \AA$ and $6962 \AA$ obtained from the campaign ${ }^{3}$ are cross-correlated with respect to the $1300 \AA$ UV continuum lightcurve from IUE using FR+RSS

3 http://www . astronomy. ohio-state.edu/ agnwatch/n7469/1cv/ technique and the resulting delays are shown by squares in Figure 7.

\section{WAVELENGTH-DEPENDENT LAG MODELLING}

To understand the nature of the observed wavelengthdependent reverberation delay and test the compatibility with the prediction of standard accretion disc theory as outlined in Section 1, i.e., $\tau \propto \lambda^{4 / 3}$, we performed modelling using two approaches. In the first approach, shown in the top panel of Figure 9 we fit the wavelength-dependent delay using a power-law model first optimising both the normalisation and index (shown by the dotted line) and then with the index fixed at $4 / 3$ (shown by the solid line). Whilst an index of $4 / 3$ is an acceptable fit, the best-fit index is $0.89 \pm 0.09$. A similar index was noted by Starkey et al. (2017) in NGC 5548. Many earlier works (Edelson et al. 2017; McHardy et al. 2018; Cackett et al. 2018) showed that the X-ray delay is usually offset with respect to the standard disc theory prediction. Therefore, in the second approach, we fit the observed delay with an offset powerlaw (constant+powerlaw) where the offset and powerlaw normalisation are free to vary while the index is fixed to $4 / 3$. The resulting fit is shown in the bottom panel of Figure 9 . According to the best-fit model the X-ray delay is offset by $\sim 0.38$ day. Interestingly, the $1300 \AA \mathrm{UV}$ continuum delay is also offset by $\sim 0.1$ day from the best-fit prediction. Assuming the lamp-post geometry of the corona, the reverberation delays at different wavelengths are calculated with respect to X-rays and shown by blue triangles with the dotted line. Details of the calculation are provided in the next section.

\section{DISCUSSION AND CONCLUSIONS}

In this work, we fit individual IUE 1220-1970 $\AA$ spectra and extract the continuum UV lightcurves from the model fitted parameters. Using the Monte Carlo simulation-based cross-correlation techniques, we show that the $2-10 \mathrm{keV}$ Xray lightcurve from $R X T E$ lags behind the UV continuum lightcurve by $3.49 \pm 0.22$ days (Figure 3 ). However, if we filter out variability slower than 5 days from the X-ray lightcurve, the cross-correlation shows that UV variability lags the Xray variability by $0.37 \pm 0.14$ days. The UV lag is consistent with the same value for both filtered and unfiltered UV lightcurves. Such a delay timescale is consistent with the light travel time from the X-ray emitting corona to the UV emitting region in the accretion disc and therefore fully consistent with the accretion disc reprocessing scenario. Therefore, UV continuum variability, from hours to weeks timescale is mainly driven by the short-term, large $\mathrm{X}$-ray variability.

\subsection{Evidence for finite size and temperature gradient in the reprocessing region}

Between the $1315 \AA$ and $1825 \AA$ UV continuum lightcurves, we show (Figure 6 ) that slower $(>5 \mathrm{~d})$ variability is delayed $(0.29 \pm 0.06$ day $)$ while the faster $(<5 \mathrm{~d})$ variability is not $(0.04 \pm 0.12$ day $)$. This is consistent with the accretion disc 
Swift Monitoring of NGC 7469
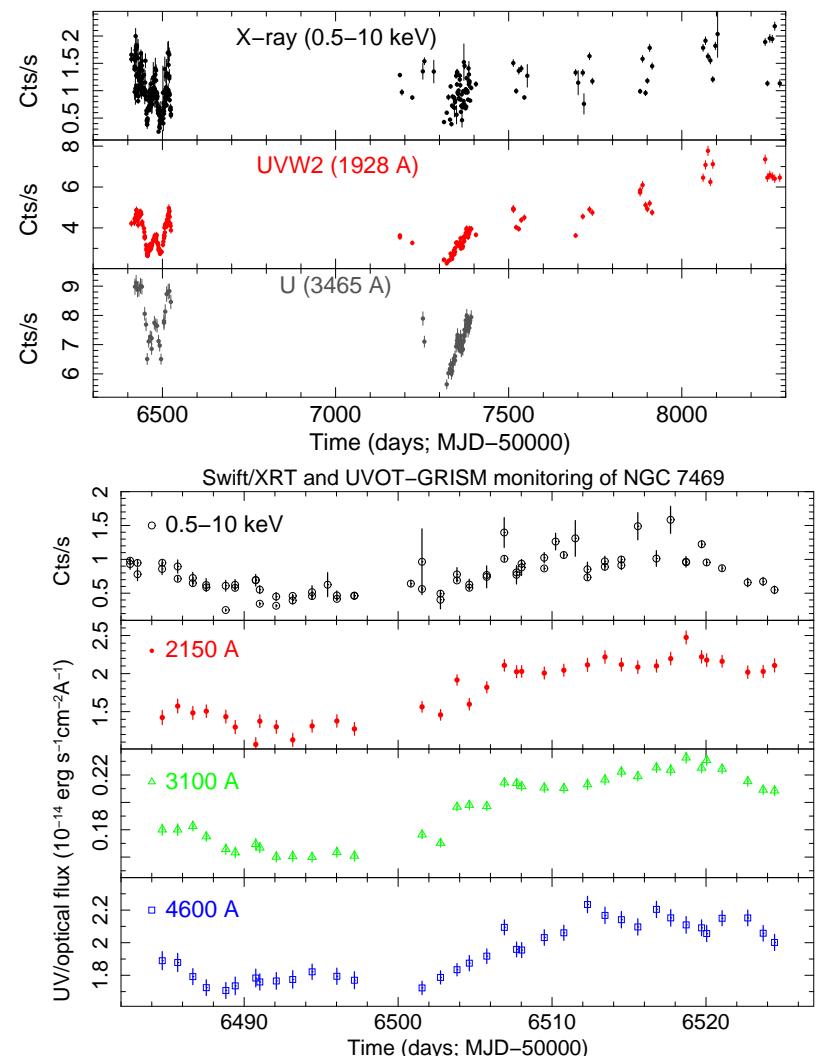

Swift Monitoring of NGC 7469
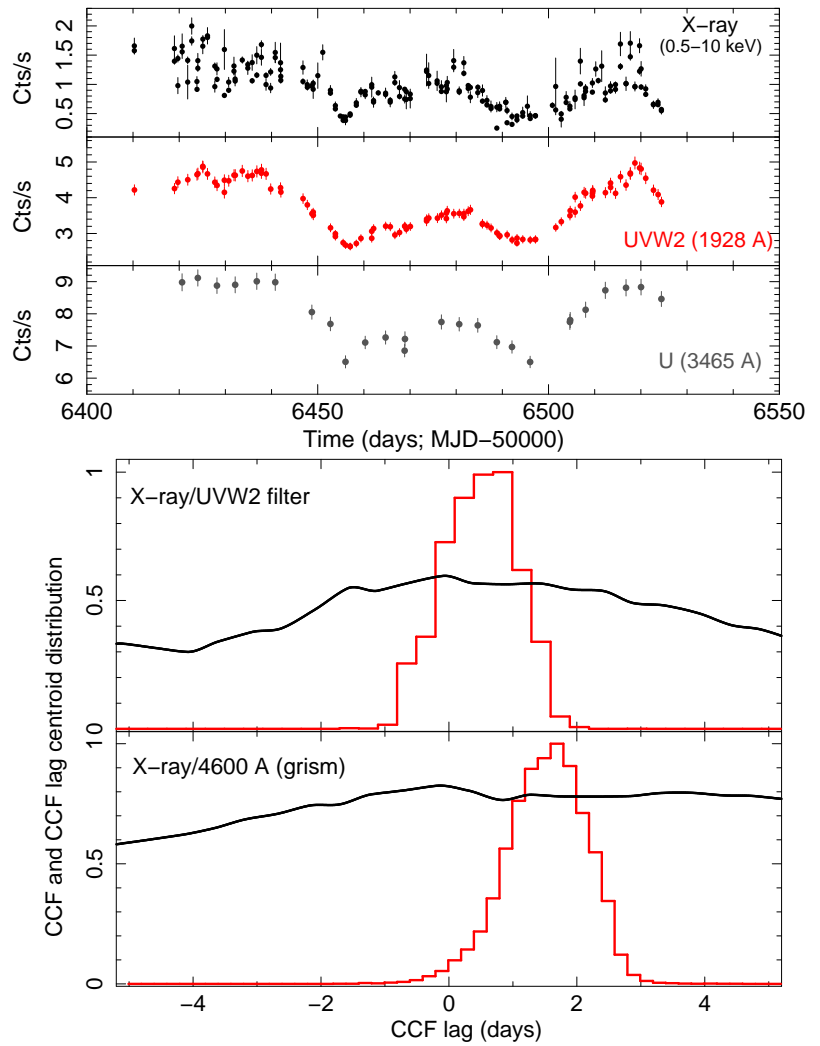

Figure 7. Reverberation delay measurements from X-ray to optical Top left panel shows long-term ( 6 years) lightcurve of NGC 7649 as monitored by Swift/XRT 0.5-10 keV X-ray (top) and Swift/UVOT UVW2 filter at $1928 \AA$ (middle) and U filter at $3465 \AA$ (bottom). To have a visual clarity of the correlated variability among X-ray, UVW2 and U filters, a zoomed version of the same lightcurve between MJD 56400 and MJD 56550 is shown in the top right panel. During the same Swift campaign, continuum lightcurves obtained from Swift/GRISM observations at $2150 \AA$, $3100 \AA$ and $4600 \AA$ are shown in the bottom left panel along with X-ray lightcurve. Bottom right panel shows cross correlation function and CCF centroid distribution from FR+RSS between X-ray and UVW2 filter lightcurves (top) and X-ray and $4600 \AA$ ARISM lightcurve (bottom) respectively.

origin of UV variability at a different wavelengths. Also, the auto-correlation function is broader for UV than for X-rays (Figure 4). Both results, delayed slow variations and broad $\mathrm{ACF}$, point to UV reprocessing from an extended rather than compact region. The outer region of the reprocessed area produces slower variability while for the same wavelength emission, the inner region causes faster variability. The idea that reprocessing occurs from a hotter or larger disc was provided by McHardy et al. (2014); Fausnaugh et al. (2016); Hall et al. (2018); Kammoun et al. (2019) and also supported by the microlensing observations (Morgan et al. 2010). If the delayed, reprocessed, UV continuum at a particular wavelength originates from a narrow region of the accretion disc, we would expect the lag distribution to be narrow and symmetric, and the UV ACF width to be similar to that of the X-rays.

\subsection{Role of fast X-ray variability}

Wavelength-dependent delay analysis using filtered X-ray and UV continuum lightcurves is found to be consistent with the predicted delay due to the reprocessed UV emission from the geometrically thin and optically thick accretion disc (Figure 9). The inclusion of Swift delay measurements from
NUV to optical is also consistent with the standard disc reprocessing delay, $\tau \propto \lambda^{4 / 3}$ relationship. Therefore, our study supports the hypothesis that the reverberation delay in the AGN accretion disc from NUV to optical is mostly driven by the X-ray variability faster than a week. The X-ray variability in AGN is usually associated with the size of the corona, which in turns depends upon the central black hole mass and in a sample of Seyfert 1 galaxies. Lu \& Yu (2001) showed that the excess variance of the short timescale $\mathrm{X}$ ray variability is anti-correlated with the black hole mass. Therefore, it suggests that in all AGN that show reverberation continuum delays, the UV delay is driven by the fast $\mathrm{X}$ ray variability, but the fast $\mathrm{X}$-ray variability timescale may vary depending upon the central black hole mass. However, testing such a hypothesis is beyond the scope of the present work.

\subsection{Comparison with the theoretical prediction}

We have compared the measured lags (Figure 8) with those expected following illumination of just an accretion disc by a point X-ray source located $6 R_{\mathrm{g}}$ above the spin axis of the black hole. We use the same model as McHardy et al. (2018), i.e., we derive the temperature distribution around a 
NGC 7469 accretion disc reverberation mapping

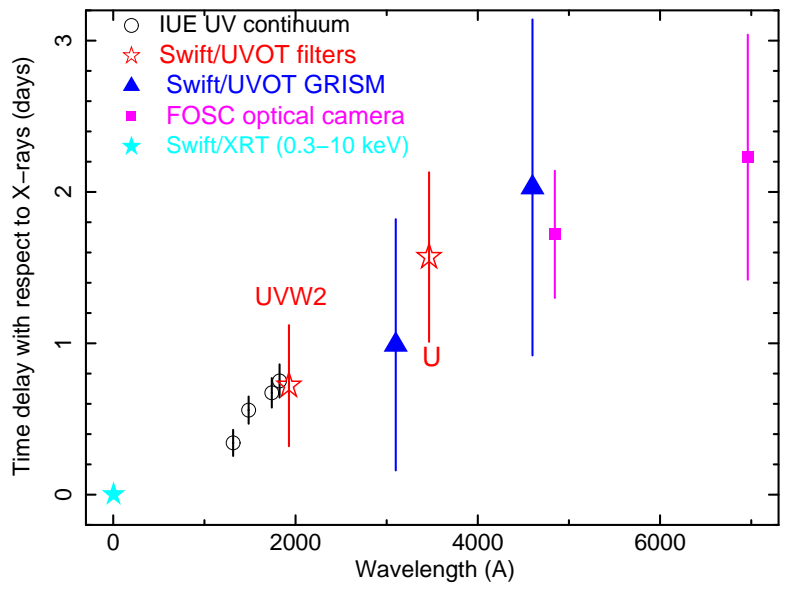

NGC 7469 flux density during different campaign

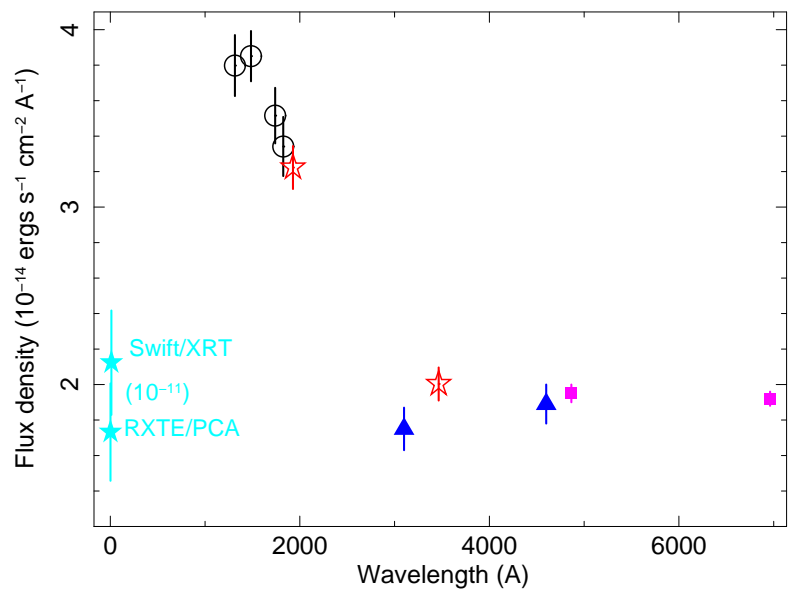

Figure 8. Reverberation delay measurements from X-ray to optical: Wavelength-dependent time delay of different UV filter and GRISM lightcurves with respect to the simultaneous X-ray lightcurve are shown by stars and triangles respectively in the left panel. For the sake of completeness, we also include delay measurements at longer wavelength performed by the FOSC spectroscopic camera on $1 \mathrm{~m}$ optical telescope at the Wise Observatory partly simultaneous with the $R X T E$ observations. Optical measurements are shown by squares. Empty circle are lag measurements using IUE continuum. Flux density measurements during different multi-wavelength campaigns are shown in the right panel. X-ray flux densities are shown in the unit of $10^{-11}$ for easy comparison. Different symbols in this panel have the same meaning as in the left panel.

black hole of a smooth accretion disc of the form described by Shakura \& Sunyaev (1973). We then illuminate the disc with X-ray impulse illumination and, taking into account the resultant change in surface temperature distribution, calculate the response in various UV and optical wavebands. The $\mathrm{X}$-ray impulse response is computed at six Swift/UVOT filter wavelengths due to the availability of their filter response curves.

As in previous work, we take the lag as the time for half of the reprocessed light to arrive (see McHardy et al. (2018) for a discussion of this point). We consider a Schwarzchild black hole and an inclination of the disc of 45 degrees. We adopt a black hole mass $9 \times 10^{6} \mathrm{M}_{\odot}$ (Peterson et al. 2014) for which $L_{\mathrm{Edd}}=1.13 \times 10^{45} \mathrm{erg} \mathrm{s}^{-1}$. We take the illuminating X-ray luminosity from the Swift/BAT 70 month survey (Baumgartner et al. 2013) of $1.8 \times 10^{43} \mathrm{ergs} / \mathrm{s}$ and multiplied it by a factor of 2 to extrapolate from the observed 14-195 keV to a broader 0.1-500 keV band. The exact value of this parameter is not critical. The accretion rate is not well known as a central starburst ring contaminates the bolometric luminosity. Values of $\dot{m} / \dot{m}_{E d d}$ between 0.05 (Mehdipour et al. 2018) and 2 (Woo and Urry 2002) have been quoted. We note that the total X-ray luminosity is then $3 \%$ of the Eddington luminosity. Assuming even a very modest X-ray to bolometric correction of a factor 10 (Netzer 2019 ), we derive an accretion rate of $\dot{m} / \dot{m}_{E d d} \sim 0.3$ and most correction factors are larger than that value. We there take $\dot{m} / \dot{m}_{E d d}=0.5$.

\subsubsection{Simulation results and inference}

In the top panel of Figure 9 we plot the model theoretical values assuming $\dot{m} / \dot{m}_{E d d}=0.5$ but also show model lines covering two extremes $\dot{m} / \dot{m}_{E d d}$ of 0.05 and 2 . Here we plot lags relative to the X-ray band. However although the lags relative to the Swift UVW2 band generally follow a smooth curve, and are similar in most AGN, the lag of the UVW2 relative to the $\mathrm{X}$-rays is usually much larger than expected from an extrapolation of the longer wavelength lags down to the X-ray band (McHardy et al. 2018). Here we note similar effects. The lag spectrum dips down below a simple powerlaw fit at wavelengths shorter than $2000 \AA$ And the model lag between the UVW2 and V-band (0.69 day) is a factor 2.3 less than the observed lag between UVW2 and V-band derived from the simple $4 / 3$ powerlaw fit to the data in Figure 9.

Discrepancy between the observed and model lag by a similar factor just within the optical bands was first noted by Collier et al. (1999) and within the UV and optical bands by McHardy et al. (2014). As we have no observed value of the V-band lag, this simple model fit is our best estimate of an observed lag. A factor of 2.3 is close to the average ratio of model to observed UVW2 to V-band lags in other AGN (McHardy et al. 2018). If we instead chose a value of $\dot{m} / \dot{m}_{E d d}=2$, the ratio between observed and model lag would drop to 1.45 and if we chose a value of $\dot{m} / \dot{m}_{E d d}=0.05$, the ratio would rise to 5 . Although the lags to the longer wavelength bands are not measured here to very high precision, nonetheless a factor of 5 discrepancy is significantly more than seen in other AGN whereas a factor of 1.45 would not be too different - assuming they are also all Schwarzchild black holes with disc inclinations and illuminating source heights similar to those assumed here, which are significant assumptions. We conclude that NGC 7469 does have a high accretion rate, nearer to 50 percent than 5 percent.

We may note that the above calculation assumes the delay due to the reprocessed emission in the continuum band is due to the X-ray-heated accretion disc. However, several works (Kotov et al. 2001; Korista and Goad 2019; Lawther et al. 2018; Chelouche et al. 2019) showed that the diffuse continuum (DC) emission from the extended BLR cloud may have significant contamination in the UVoptical continuum lag measurements, particularly close to 

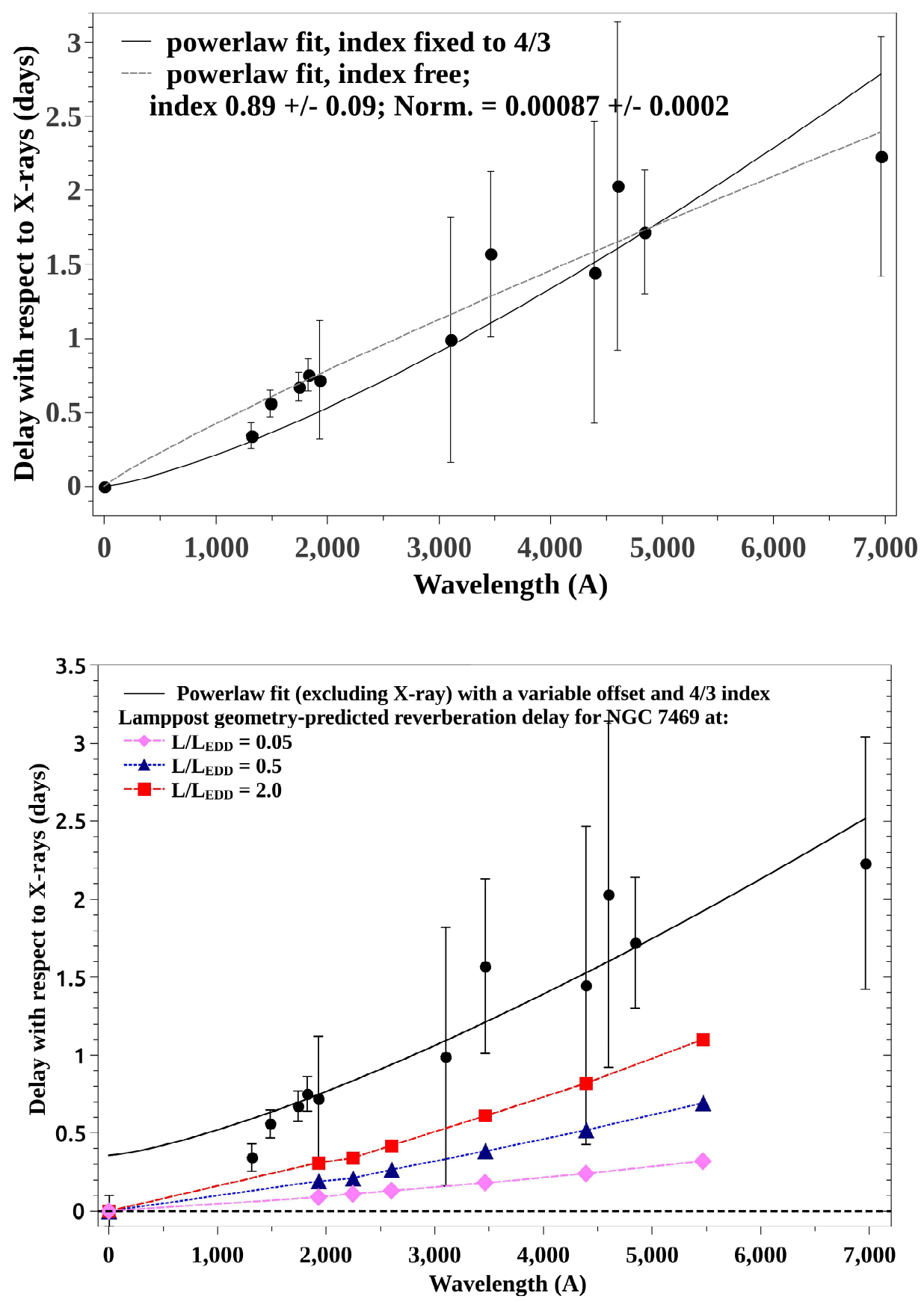

Figure 9. Reverberation delay modelling from X-ray to optical: Top panel: the fitting of wavelength-dependent delay spectrum (same spectral points as the left panel of Figure 8 but all represented by solid circles for the sake of uniformity) including X-rays using two powerlaws: with variable normalisation and index (shown by the dotted line) and with variable normalisation but fixing the index at $4 / 3$ (shown by the solid line). Bottom panel: the powerlaw fit of wavelength-dependent delay with an offset where the offset and the powerlaw normalisation are free to vary while the index is fixed to $4 / 3$. The X-ray data point is excluded while fitting and shown for clarity. The best-fit shows the X-ray and UV $(1300 \AA)$ delay measurements are below the fitted model by $\sim 0.38$ day and $\sim 0.1$ day respectively. Squares, triangles and diamonds with the dotted lines show the theoretical X-ray reverberation delay estimation with $L / L_{E D D}$ of 0.05 , 0.5 and 2.0 respectively for NGC 7469 assuming the 'lamppost' geometric configuration of the corona without any additional X-ray offset.

the Balmer continuum. The detail study of the DC contribution to the observed lag in NGC 7469 is beyond the scope of the present work.

\section{ACKNOWLEDGEMENT}

We thank the referee for comments and suggestions which help to improve the clarity of the paper. MP acknowledges Royal Society-SERB Newton International Fellowship sup- 
port funded jointly by the Royal Society, UK and the Science and Engineering Board of India (SERB) through NewtonBhabha Fund. IMcH acknowledges support from a Royal Society Leverhulme Trust Research Fellowship LT160006 and from STFC grant ST/M001326/1. EMC gratefully acknowledges support from the National Science Foundation through award number AST-1909199. KH acknowledges support from STFC grant ST/R000824/1.

\section{REFERENCES}

Arev́alo P. Uttley P., 2006, MNRAS, 367, 801

Arev́alo, P., Uttley, P., Kaspi, S., Breedt, E., Lira, P., McHardy, I. M., 2008, MNRAS , 389, 1479

Arev́alo, P., Uttley, P., Lira, P., Breedt, E., McHardy, I. M., Churazov, E. 2009, MNRAS, 397, 2004

Ayres, T. R. 1993, PASP, 105, 538

Baldi, R. D., Behar, E., Laor, A., Horesh, A. 2015, MNRAS, 454, 4277

Baldwin, J., Ferland, G., Korista, K., Verner, D., 1995, ApJ, 455, L119

Baumgartner, W. H., Tueller, J., Markwardt, C. B., Skinner, G. K., Barthelmy, S., Mushotzky, R. F., Evans, P. A., Gehrels, N. 2013, ApJS, 207, 19

Bentz, M. C. and Katz, S. 2015, PASP, 127, 67

Bentz, M. C., Walsh J. L., Barth A. J. et al., 2009, ApJ, 705, 199

Blandford R. D. and McKee C. F. 1982, ApJ, 255, 419 ve01

Blustin, A. J.; Kriss, G. A.; Holczer, T.; Behar, E.; Kaastra, J. S.; Page, M. J.; Kaspi, S.; Branduardi-Raymont, G.; Steenbrugge, K. C. 2007, A\&A, 466, 107

Brandt, W. N., Fabian, A. C., Nandra, K. et al. 1993, MNRAS, 265, 996

Breedt, E., McHardy, I. M., Arev́alo, P., Uttley, P., Sergeev, S. G., Minezaki, T., Yoshii, Y., Sakata, Y., Lira, P., Chesnok, N. G. 2010, MNRAS, 403, 605

Breedt, E., Arev́alo, P., McHardy, I. M.; Uttley, P.; Sergeev, S. G.; Minezaki, T. et al. 2009, MNRAS, 394, 427

Cackett, E. M., Chiang, Chia-Ying, McHardy, I., Edelson, R., Goad, M. R., Horne, K., Korista, K. T. 2018, ApJ, 857, 53

Cackett E. M., Horne K. and Winkler H. 2007, MNRAS, 380, 669

Cameron, D. T., McHardy, I. M., Dwelly, T., Breedt, E., Uttley, P., Lira, P., Arevalo, P., 2012, MNRAS, 422, 902

Chelouche, D., Pozo, N., Francisco, K. S. 2019, NatAs, 3, 251

Cleveland, W. S. \& Devlin, S. J., 1988, "Locally weighted regression: An approach to regression analysis by local fitting", Journal of the American Statistical Association, 83, 596

Collier S. J., Horne K., Wanders I. and Peterson B. M., 1999, MNRAS, 302, L24

Collier, S. J., Horne, K., Kaspi, S., Netzer, H., Peterson, B. M., Wanders, I. et al. 1998, ApJ, 500, 162

Dai, X., Kochanek, C. S., Chartas, G., Kozlowski, S., Morgan, C. W., Garmire, G., Agol, E., 2010, ApJ , 709, 278

Dexter, J. and Agol, E ., 2011, ApJ, 727, L24

Epanechnikov, V. A. 1969, Theory Probab. Appl., 14, 153, doi:10.1137/1114019

Edelson R., et al., 2015, ApJ, 806, 129

Edelson R., et al., 2017, ApJ, 840, 41

Edelson, R., Gelbord, J., Cackett, E., Peterson, B. M., Horne, K., Barth, A. J. et al. 2019, ApJ, 870, 123

Fausnaugh M. M., et al., 2016, ApJ, 821, 56

Gardener, E. and Done, C. 2017, MNRAS, 470, 3591

Hall, P. B., Sarrouh, G. T. and Horne, K. 2018, ApJ, 854, 93

Kammoun, E.S. et al. 2019, ApJL, 879, 24

Kaspi, S., Ibbetson, P. A., Mashal, E., \& Brosch, N. 1996, Wise Obs. Tech. Rep., No. 6

Korista, K. T. and Goad, M. R. 2019, eprint arXiv:1908.07757
Korista, K. T. and Goad, M. R., 2001, ApJ, 553, 695

Korista, K. T. and Goad, M. R., 2000, ApJ, 536, 284

Kotov O., Churazov E., Gilfanov M. , 2001, MNRAS , 327, 799

Kriss, G. A., Blustin, A., Branduardi-Raymont, G., Green, R. F., Hutchings, J., Kaiser, M. E. 2003, A\&A, 403, 473

Kriss, G. A., Peterson, Bradley M., Crenshaw, D. M., Zheng, W. 2000, ApJ, 535, 58

Lawther, D., Goad, M. R., Korista, K. T., Ulrich, O., Vestergaard, M. 2018, MNRAS, 481, 533

Lu, Y. and Yu, Q., 2001, MNRAS, 324, 653

Marshall, K., Ryle, W. T., Miller, H. R., 2008, ApJ, 677, 880

Marshall, N., Warwick, R. S., Pounds, K. A. 1981, MNRAS, 194, 987

McHardy, I. M., Connolly, S. D., Horne, K., Cackett, E. M., Gelbord, J., Peterson, B. M., Pahari, M., Gehrels, N. et al. 2018, MNRAS, 480, 2881

McHardy, I. M., et al., 2016, Astronomische Nachrichten, 337, 500

McHardy, I. M., et al., 2014, MNRAS, 444, 1469

McHardy I. M., Papadakis I. E., Uttley P., Page M. J., Mason K. O., 2004, MNRAS , 348, 783

McHardy, I. M., Uttley, P., Mason, K., Page, M. 2003, ASPC, 290,111

Mehdipour, M., Kaastra, J. S., Costantini, E., Behar, E., Kriss, G. A., Bianchi, S. et al. 2018, A\&A, 615, 72

Morgan C. W., Kochanek C. S., Morgan N. D., Falco E. E., 2010, ApJ, 712, 1129

Mosquera, A. M., Kochanek, C. S., Chen, B., Dai, X., Blackburne, J. A., Chartas, G., 2013, ApJ, 769, 53

Nandra, K., Clavel, J., Edelson, R. A., George, I. M., Malkan, M. A., Mushotzky, R. F. et al. 1998, ApJ, 505, 594

Narayan, R. 1996, ApJ, 462, 136

Netzer, H. 2019, MNRAS, 488, 5185

Nichols, J. S., Garhart, M. P., de la Pena, M. D., \& Levay, K. L. 1993, NEWSIPS Information Manual : Low-Dispersion Data v1.0, CSC/SD-93/6062

Noda H. et al., 2016, ApJ, 828, 78

Pal, M. and Naik, S. 2018, MNRAS, 414, 5351

Pal, M., Dewangan, G. C., Connolly, S., Misra, R. 2017, MNRAS, 466, 1777

Papadakis I. E., Nandra K., Kazanas D., 2001, ApJ , 554, 133

Peterson, B. M., Grier, C. J., Horne, K., Pogge, R. W., Bentz, M. C., De Rosa, G. et al. 2014, ApJ, 795, 149

Peterson B. M. 2014, SSRv, 183, 253

Peterson, B. M. et al. 2004, ApJ, 613, 682

Peterson, B. M., Wanders, I., Horne, K., Collier, S., Alexander, T., \& Kaspi, S. 1998, PASP, 110, 660

Petrucci, P. O., Maraschi, L., Haardt, F., Nandra, K. 2004, A\&A, 413, 477

Poole, T. S., Breeveld, A. A., Page, M. J., Landsman, W. et al. 2008, MNRAS, 383, 627

Pronik, I. I., Metik, L. P., Merkulova, N. I. 1997, A\&A, 318, 721

Salamanca, I., Alloin, D., \& Pelat, D. 1995, A\&AS, 111, 283

Seifina, E., Titarchuk, L., Ugolkova, L. 2018, A\&A, 619, 21

Shakura, N. I.\& Sunyaev, R. A., 1973, A\&A, 24, 337

Shemmer, O., Uttley, P., Netzer, H., McHardy, I. M., 2003, MNRAS, 343, 1341

Shapovalova, A. I., Popovic, L. C., et al. 2017, MNRAS, 466, 4759

Shappee B. J., et al., 2014, ApJ, 788, 48

Shull, J., Michael, S., Matthew, D., Charles W. 2012, ApJ, 752, 162

Seyfert, C. K. 1943, ApJ, 97, 28

Starkey, D., Horne, Keith, Fausnaugh, M. M., Peterson, B. M., Bentz, M. C., Kochanek, C. S. et al. 2017, ApJ, 835, 65

Sun et al. 2018, ApJ, 857, 86

Troyer, J., Starkey D., Cackett E. M., Bentz M. C., Goad M. R.,Horne K., Seals J. E., 2016, MNRAS, 456, 4040

Uttley, P., Edelson, R., McHardy, I. M., Peterson, B. M., Markowitz, A. 2003, ApJ, 584, L53 
Uttley, P., Wilkinson, T., cassatella, P. et al. 2011, MNRAS, 414, L60

Vestergaard, M. and Wilkes, B. J. 2001, ApJSS, 134, 1

Wanders, I., Peterson, B. M., Alloin, D., Ayres, T. R., Clavel, J., Crenshaw, D. M. et al. 1997, ApJ, 113, 69

Welsh W. F., 1999, PASP, 111, 1347

Welsh, W. F., Peterson, B. M., Koratkar, A. P., Korista, K. T. 1998, ApJ, 509, 118

White, R. J. \& Peterson, B. M. 1994, PASP, 106, 879

Woo, Jong-Hak and Urry, C. M. 2002, ApJ, 579, 530

Zu, Ying, Kochanek, C. S., Peterson, Bradley M., 2011, ApJ, 735, 80

This paper has been typeset from a $\mathrm{T}_{\mathrm{E}} \mathrm{X} / \mathrm{L}_{\mathrm{A}} \mathrm{T}_{\mathrm{E}} \mathrm{X}$ file prepared by the author. 